\title{
Exploring the geometry of qutrit state space using symmetric informationally complete probabilities
}

\author{
Gelo Noel M. Tabia ${ }^{1,2, *}$ and D. M. Appleby ${ }^{1,3, \dagger}$ \\ ${ }^{1}$ Perimeter Institute for Theoretical Physics, 31 Caroline Street North, Waterloo, Ontario, Canada, N2L 2 Y5 \\ ${ }^{2}$ Department of Physics and Astronomy and Institute for Quantum Computing, \\ University of Waterloo, 200 University Avenue West, Waterloo, Ontario, Canada, N2L 3G1 \\ ${ }^{3}$ Stellenbosch Institute for Advanced Study, Wallenberg Research Centre at \\ Stellenbosch University, Marais Street, Stellenbosch, South Africa, 7600
}

\begin{abstract}
We examine the geometric structure of qutrit state space by identifying the outcome probabilities of symmetric informationally complete (SIC) measurements with quantum states. We categorize the infinitely many qutrit SICs into 8 SIC-families corresponding to independent orbits of the extended Clifford group. Every SIC can be uniquely identified from a set of geometric invariants that we use to establish several properties of the convex body of qutrits, which include a simple formula describing its extreme points, an expression for the rotation between the probability vectors for distinct qutrit SICs, and a polar equation for its boundary states.
\end{abstract}

PACS numbers: 03.65.Aa, 03.65.Wj, 04.60.Pp

\section{INTRODUCTION}

In quantum mechanics, the state of a physical system is usually described by a density operator, which is a positive semidefinite, Hermitian matrix with unit trace. For any pair of Hermitian matrices $A$ and $B$, define the Hilbert-Schmidt inner product $\langle A, B\rangle_{\mathrm{HS}}=\operatorname{Tr}(A B)$. The space of Hermitian matrices $\operatorname{Herm}\left(\mathcal{H}^{d}\right)$ on a Hilbert space $\mathcal{H}^{d}$ then forms a Euclidean space. If we consider the set of density operators $\mathcal{D}\left(\mathcal{H}^{d}\right)$ as a subset of $\operatorname{Herm}\left(\mathcal{H}^{d}\right)$, then we can think of $d$-dimensional quantum states as points in a $\left(d^{2}-1\right)$-dimensional convex set $\mathcal{C} \subset \mathbb{R}^{d^{2}}$ that is isomorphic to $\mathcal{D}\left(\mathcal{H}^{d}\right)$. We expect the geometric features of the convex set $\mathcal{C}$ to reflect properties of density operators. For example, the full geometry of $\mathcal{C}$ is well-known for $d=2$; it is a solid 3-dimensional ball called the Bloch ball. The spherical boundary of the ball corresponds to pure states, where orthogonal states get mapped onto antipodal points, and interior points correspond to mixtures, each of which can be decomposed into any convex combination of pure states whose convex hull contains that point. We can also compare how similar any two states $\rho_{1}$ and $\rho_{2}$ are by measuring their HilbertSchmidt distance $D_{\mathrm{HS}}\left(\rho_{1}, \rho_{2}\right)=\sqrt{\operatorname{Tr}\left(\rho_{1}-\rho_{2}\right)^{2}}$. Little, however, is known of the same convex geometry for quantum states in higher dimensions. Much effort has been made in uncovering the rich, intricate structure of $\mathcal{C}$ for $d=3$ by examining the various 2 - and 3 -dimensional sections obtained from the generalized Bloch representation for qutrits [1 5] but many details of its overall structure remain unknown.

In this paper, we analyze the geometric features of qutrits in terms of the probabilities for a special measurement called a symmetric informationally complete

\footnotetext{
* Corresponding author: gtabia@perimeterinstitute.ca

$\dagger$ mappleby@perimeterinstitute.ca
}

(SIC) measurement 6 12. A SIC measurement maps each density operator into a unique probability vector, which represents one way of specifying an isomorphism of $\mathcal{D}\left(\mathcal{H}^{d}\right)$ onto a subset of $\mathbb{R}^{d^{2}}$. This particular mapping allows us to characterize quantum states as a proper subset of the probability simplex, where the restriction is imposed mainly by a special version of the Born rule.

We find that the SIC probabilities provide us with a novel way of characterizing properties of qutrits, particularly with respect to the flat geometry induced by the Euclidean metric defined on the simplex. Here we present three main results:

(i) The extreme points representing pure states for qutrits are obtained from a simple formula that picks out a submanifold of points lying on a certain sphere.

(ii) The probability vectors obtained for any pair of SICs are related by a rotation with a very simple form, which we construct explicitly. It may be worthwhile to note that the rotation does not necessarily arise from a unitary transformation between the SICs involved (except when the SICs are unitarily equivalent).

(iii) The boundary points are described using a polar equation that gives their radial distances from the uniform distribution, which represents the maximally mixed state. This is different from existing methods that analyze the geometry of qutrits by studying the boundary of various 2-dimensional sections [1, 4, 5].

The motivation for such a study is twofold. Firstly, the geometry of quantum states is interesting in its own right and a better understanding of it may have important repercussions for various applications of quantum information processing. Secondly, Fuchs and Schack advocate a framework for reformulating quantum mechanics directly in terms of probabilities without mentioning 
Hilbert space at all 13. A better understanding of the structure of SIC probabilities may prove useful in identifying the basic axioms needed for reconstructing quantum theory exclusively in terms of probabilities.

\section{PROPERTIES OF WEYL-HEISENBERG QUTRIT SICS}

One way to represent quantum states in terms of probabilities is to express density operators in terms of $d^{2}$ linearly independent projectors $\Pi_{i}=\left|\psi_{i}\right\rangle\left\langle\psi_{i}\right|$ such that

$$
\left|\left\langle\psi_{i} \mid \psi_{j}\right\rangle\right|^{2}=\frac{d \delta_{i j}+1}{d+1}
$$

When each projection is scaled by $\frac{1}{d}$, we get a measurement called a symmetric informationally complete positive operator-valued measurement (SIC-POVM), a topic of considerable interest in the quantum physics community. In this paper, the set $\left\{\Pi_{i}\right\}_{i=1}^{d^{2}}$ is called a $S I C$ for short.

The one-to-one correspondence between the outcome probabilities $p(i)$ of a SIC-POVM and density operators is given by 14

$$
\rho=\sum_{i=1}^{d^{2}}\left[(d+1) p(i)-\frac{1}{d}\right] \Pi_{i} .
$$

Thus, the SIC probability vectors $\vec{p}$ provide an equivalent description of quantum states, which we call the $S I C$ representation. It follows that we can always choose the coordinates of $\mathcal{C}$ such that $\vec{p} \in \mathcal{C}$ for all $\vec{p}$ associated with a quantum state according to Eq. (2). The relation between the Hilbert-Schmidt inner product for a pair of density operators $\rho_{1}$ and $\rho_{2}$, and the scalar product of their respective probability vectors $\vec{p}_{1}$ and $\vec{p}_{2}$ is given by

$$
\operatorname{Tr}\left(\rho_{1} \rho_{2}\right)=d(d+1) \vec{p}_{1} \cdot \vec{p}_{2}-1 \text {. }
$$

All SICs constructed to date have a certain group covariance property. Let $G$ be a group of $d^{2}$ elements and let $g \mapsto U_{g}$ be a projective representation of $G$ on $\mathcal{H}^{d}$. Let $|\psi\rangle \in \mathcal{H}^{d}$. If the set of vectors $U_{g}|\psi\rangle$ generates a SIC $\mathcal{S}$, we say that $\mathcal{S}$ is covariant with respect to $G$. The seed vector $|\psi\rangle$ for $\mathcal{S}$ is called a fiducial vector.

In almost all known cases, the unitaries that produce SICs belong to the Weyl-Heisenberg group. Let $\{|j\rangle\}_{j=0}^{d-1}$ be an orthonormal basis for $\mathcal{H}^{d}$. The Weyl-Heisenberg group is generated by the shift $X$ and phase $Z$ operators,

$$
\begin{aligned}
& X|j\rangle=|j+1 \quad \bmod d\rangle, \\
& Z|j\rangle=\omega^{j}|j\rangle
\end{aligned}
$$

where $\omega=e^{i \frac{2 \pi}{d}}$. We can act with powers of $X$ and $Z$ on a SIC fiducial $|\psi\rangle$ so that the resulting vectors

$$
\left|\psi_{m n}\right\rangle=X^{m} Z^{n}|\psi\rangle, \quad m, n=0,1, \ldots, d-1,
$$

satisfy Eq. (1). In that case, the projectors associated with $\left|\psi_{m n}\right\rangle$ form a SIC, which we call a Weyl-Heisenberg SIC.

The operators $X$ and $Z$ generate the group

$$
W(d)=\left\{\omega^{\alpha} X^{m} Z^{n} \mid \alpha, m, n=0,1, \ldots, d-1\right\},
$$

which we call the Weyl-Heisenberg group. Note that $W(d)$ is of order $d^{3}$. However, two unitaries which differ only by a phase generate the same SIC projector, so the SIC itself only contains $d^{2}$ elements.

The normalizer of the Weyl-Heisenberg group is called the Clifford group $C(d)$ [9, 15 17, which is itself a unitary subgroup in dimension $d$. If $U \in C(d)$ is a Clifford unitary operator and $W(d)$ is the Weyl-Heisenberg group then

$$
U W(d) U^{\dagger}=W(d)
$$

If the set of antiunitary operators that map $W(d)$ to itself are included, we get the extended Clifford group.

In $d=3$, we have

$$
X=\left(\begin{array}{lll}
0 & 0 & 1 \\
1 & 0 & 0 \\
0 & 1 & 0
\end{array}\right), \quad Z=\left(\begin{array}{ccc}
1 & 0 & 0 \\
0 & \omega & 0 \\
0 & 0 & \omega^{2}
\end{array}\right)
$$

As a matter of convention, we label the Weyl-Heisenberg SIC projectors $\Pi_{i}$ with index $i=d m+n+1$, so we have $i=1,2, \ldots, 9$ for qutrit SICs. For example, $\Pi_{6}$ is the SIC projector corresponding to $\left|\psi_{6}\right\rangle=X Z^{2}|\psi\rangle$.

Every Weyl-Heisenberg SIC in $d=3$ can be obtained by acting with an (extended) Clifford (anti)unitary on a $\mathrm{SIC}$ with fiducial vector

$$
\left|\psi_{t}\right\rangle=\frac{1}{\sqrt{2}}\left(\begin{array}{c}
0 \\
1 \\
-e^{2 i t}
\end{array}\right), \quad t \in\left[0, \frac{\pi}{6}\right]
$$

Fiducials corresponding to distinct values of $t$ in the range $\left[0, \frac{\pi}{6}\right]$ generate distinct orbits of the extended Clifford group. In Ref. 9] it is shown that there are 3 types of orbits of the extended Clifford group in $d=3$ for which $\left|\psi_{t}\right\rangle$ is in the orbit: the infinitely many generic ones for $t \in\left(0, \frac{\pi}{6}\right)$ and 2 exceptional ones for the endpoints $t=0$ and $t=\frac{\pi}{6}$.

In the generic case, each extended Clifford orbit consists of 8 SICs generated by the fiducial vectors:

$$
\begin{aligned}
\left|\psi_{t}^{(0 \pm)}\right\rangle & =\frac{1}{\sqrt{2}}\left(\begin{array}{c}
0 \\
e^{\mp i t} \\
-e^{ \pm i t}
\end{array}\right) \\
\left|\psi_{t}^{(\eta \pm)}\right\rangle & =\sqrt{\frac{2}{3}}\left(\begin{array}{c}
\omega^{\eta} \sin t \\
\sin \left(t \pm \frac{2 \pi}{3}\right) \\
\sin \left(t \mp \frac{2 \pi}{3}\right)
\end{array}\right)
\end{aligned}
$$


For $t=\frac{\pi}{6}$, there are 4 distinct SICs whose fiducials can be chosen as

$$
\begin{aligned}
\left|\psi_{\frac{\pi}{6}}^{(0)}\right\rangle=\frac{1}{\sqrt{2}}\left(\begin{array}{c}
0 \\
1 \\
1
\end{array}\right), & \left|\psi_{\frac{\pi}{6}}^{(1)}\right\rangle=\frac{1}{\sqrt{6}}\left(\begin{array}{c}
\omega \\
1 \\
-2
\end{array}\right), \\
\left|\psi_{\frac{\pi}{6}}^{(2)}\right\rangle=\frac{1}{\sqrt{6}}\left(\begin{array}{c}
\omega^{2} \\
1 \\
-2
\end{array}\right), & \left|\psi_{\frac{\pi}{6}}^{(3)}\right\rangle=\frac{1}{\sqrt{6}}\left(\begin{array}{c}
1 \\
1 \\
-2
\end{array}\right) .
\end{aligned}
$$

For $t=0$, the fiducial generating the unique SIC can be chosen as

$$
\left|\psi_{0}\right\rangle=\frac{1}{\sqrt{2}}\left(\begin{array}{c}
0 \\
1 \\
-1
\end{array}\right) .
$$

It is worth mentioning here that the SICs of Eq. (10) are inequivalent with respect to Clifford unitaries; however, some of the SICs for different values of $t$ are still related by a unitary operator that is not a member of the Clifford group. Specifically, Zhu [18 has shown that the SICs for $t, \frac{\pi}{9}-t$, and $\frac{\pi}{9}+t$ are, in fact, unitarily equivalent to each other, with the unitary transformation relating them being

$$
U=\operatorname{diag}\left(1, u, u^{2}\right), \quad u=e^{-i \frac{2 \pi}{9}},
$$

which is not a Clifford unitary. Moreover, there are no other unitary equivalences. This means that every pair of SICs on any two different orbits corresponding to $t \in$ $\left[0, \frac{\pi}{18}\right]$ are not equivalent.

Associated with each value of $t$ are two sets of closely related geometric quantities. The first set consists of the traces of the product of three SIC projectors called triple products $T_{i j k}$,

$$
T_{i j k}=\operatorname{Tr}\left(\Pi_{i} \Pi_{j} \Pi_{k}\right) .
$$

It is shown in Ref. 19 that two SICs are unitarily equivalent if and only if the triple products are the same, up to permutation. The other set consists of the structure coefficients $S_{i j k}$ that describe multiplication between SIC projectors,

$$
\Pi_{i} \Pi_{j}=\sum_{k} S_{i j k} \Pi_{k} .
$$

It is straightforward to show that the structure coefficients can be obtained from the triple products in the following way:

$$
S_{i j k}=\frac{1}{d}\left[(d+1) T_{i j k}-\frac{d \delta_{i j}+1}{d+1}\right] .
$$

In Sec. III we shall see that the real parts $\tilde{T}_{i j k}=$ $\operatorname{Re}\left[T_{i j k}\right]$ and $\tilde{S}_{i j k}=\operatorname{Re}\left[S_{i j k}\right]$ of the triple products and structure coefficients, respectively, are adequate for describing probability vectors corresponding to quantum states.
It is easy to compute $S_{i j k}$ when some of the indices are identical:

$$
S_{i i i}=1, \quad S_{i j j}=S_{j i j}=\frac{1}{4}, \quad S_{j j i}=0 .
$$

It is also straightforward to compute $\tilde{S}_{i j k}$ for $i \neq j \neq k$ using Eq. 14) and Eq. (16). Taking only the real parts, the distinct nonzero values are $-\frac{1}{4}$ and 3 other values we denote as $x_{t}, y_{t}$, and $z_{t}$ :

$$
\begin{aligned}
& x_{t}=-\frac{1}{6}\left(\cos 6 t+\frac{1}{2}\right), \\
& y_{t}=-\frac{1}{6}\left[\cos \left(6 t+\frac{2 \pi}{3}\right)+\frac{1}{2}\right] \equiv x_{\frac{\pi}{9}+t}, \\
& z_{t}=-\frac{1}{6}\left[\cos \left(6 t-\frac{2 \pi}{3}\right)+\frac{1}{2}\right] \equiv x_{\frac{\pi}{9}-t} .
\end{aligned}
$$

It can be seen that SICs with parameter values $t, \frac{\pi}{9}-t$, and $\frac{\pi}{9}+t$ have the same values of $x, y$, and $z$, up to a permutation - confirming the fact mentioned earlier, that such SICs are unitarily equivalent.

Hughston [20] has shown that the SIC vectors of the single SIC for $t=0$ can be obtained from the inflection points of a family of cubic elliptic curves on the complex projective plane known as the Hesse pencil (see also Bengtsson [21.) There are 8 SICs with parameter value $t=\frac{\pi}{9}$ that are unitarily equivalent to the single SIC for $t=0$, and we call these 9 SICs the Hesse SICs. The particular SIC specified by Eq. 12 shall be called the canonical Hesse SIC.

For the canonical Hesse SIC, Eq. (18) gives

$$
x_{0}=-\frac{1}{4}, \quad y_{0}=z_{0}=0 .
$$

It is the simplicity of these numbers that leads to an elegant characterization of qutrit pure states in Sec. III.

There are some simple rules for finding the index triples corresponding to the values $-\frac{1}{4}, x_{t}, y_{t}$, and $z_{t}$, which we describe next.

To each qutrit SIC-family $\left|\psi_{t}^{(\eta \pm)}\right\rangle(\eta=0,1,2,3)$ in Eq. 10 we assign an index generator $G_{\eta \pm}$ that helps us choose the index triples $(i j k)$ for each distinct value of $\tilde{S}_{i j k}$. They are listed in Table I. To illustrate what the rules are, let us take the SIC $\left\lceil\psi_{t}^{(2+)}\right\rangle$ as a specific example.

For $\tilde{S}_{i j k}=-\frac{1}{4}$, take the index triples on the same row. Looking at $G_{2+}$ in Table I, we see that the relevant set of $(i j k)$ for $\left|\psi_{t}^{(2+)}\right\rangle$ is

$$
\{(168),(249),(357)\}
$$

and all permutations of indices for each $(i j k)$.

For $\tilde{S}_{i j k}=x_{t}$, take the index triples belonging to the same column, or those on entirely different rows and columns. Thus, the relevant set of $(i j k)$ from $G_{2+}$ is

$\{(123),(645),(897),(147),(693),(825),(195),(627),(843)\}$ 
TABLE I. Structure coefficient index generators for qutrit SICs. The rules for choosing the index triples $(i j k)$ for each distinct value of $\tilde{S}_{i j k}$ are described in the main text.

$$
\begin{array}{lll}
G_{0+}=\left[\begin{array}{lll}
1 & 2 & 3 \\
4 & 5 & 6 \\
7 & 8 & 9
\end{array}\right], & G_{0-}=\left[\begin{array}{lll}
1 & 3 & 2 \\
4 & 6 & 5 \\
7 & 9 & 8
\end{array}\right], \\
G_{1+}=\left[\begin{array}{lll}
1 & 5 & 9 \\
2 & 6 & 7 \\
3 & 4 & 8
\end{array}\right], & G_{1-}=\left[\begin{array}{lll}
1 & 9 & 5 \\
2 & 7 & 6 \\
3 & 8 & 4
\end{array}\right], \\
G_{2+}=\left[\begin{array}{lll}
1 & 6 & 8 \\
2 & 4 & 9 \\
3 & 5 & 7
\end{array}\right], & G_{2-}=\left[\begin{array}{lll}
1 & 8 & 6 \\
2 & 9 & 4 \\
3 & 7 & 5
\end{array}\right], \\
G_{3+}=\left[\begin{array}{lll}
1 & 4 & 7 \\
2 & 5 & 8 \\
3 & 6 & 9
\end{array}\right], & G_{3-}=\left[\begin{array}{lll}
1 & 7 & 4 \\
2 & 8 & 5 \\
3 & 9 & 6
\end{array}\right] .
\end{array}
$$

and all permutations of indices for each $(i j k)$.

For $\tilde{S}_{i j k}=y_{t}$, take the index triples such that the first two indices belong to the same column, and the last one is in a different row and belongs to the succeeding column when counting in a cyclic manner. By succeeding we mean that "column 2 is after column 1 ", "column 3 is after column 2", and "column 1 is after column 3." Thus, the relevant set of $(i j k)$ from $G_{2+}$ is

$$
\{(125),(647),(893),(236),(458),(971),(314),(569),(782)\}
$$

and all permutations of indices for each $(i j k)$.

For $\tilde{S}_{i j k}=z_{t}$, we have a similar rule as in $y_{t}$ but take the last index from the preceding column. Thus, the relevant set $(i j k)$ from $G_{2+}$ is

$\{(127),(643),(895),(238),(451),(976),(319),(562),(784)\}$ and all permutations of indices for each $(i j k)$.

Any other index triple $(i j k)$ not specified above has $\tilde{S}_{i j k}=0$.

We do not need them in this paper but it is possible to construct similar, though somewhat more complicated, rules for getting the imaginary parts of $S_{i j k}$.

\section{PURE STATES IN THE SIC REPRESENTATION}

Since quantum state space is a compact convex body $\mathcal{C}$ in $\mathbb{R}^{d^{2}}$, the Krein-Milman theorem 22] states that it is equal to the convex hull of its extreme points, which are the pure states. It is therefore natural to ask what are the conditions on SIC probability vectors $\vec{p}$ such that they correspond to pure states. In terms of density operators, a pure state is represented by a rank-1 projector, $\rho^{2}=\rho$.

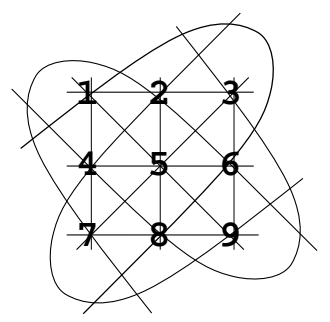

FIG. 1. The 12 lines of a finite affine plane over the Galois field $\mathrm{GF}(3)$ representing the index triples $(i j k) \in Q$ in Eq. 28. The indices are depicted as 9 points and each line contains 3 points as marked.

A remarkable theorem [23, 24] states that for a hermitian operator $\rho=\rho^{\dagger}$, an equivalent condition for defining a pure state is given by

$$
\operatorname{Tr}\left(\rho^{2}\right)=\operatorname{Tr}\left(\rho^{3}\right)=1 .
$$

Using the SIC representation of $\rho$ given by Eq. (2), Eq. 20 becomes 25.

$$
\begin{aligned}
\sum_{i} p(i)^{2} & =\frac{2}{d(d+1)}, \\
\sum_{i, j, k} T_{i j k} p(i) p(j) p(k) & =\frac{d+7}{(d+1)^{3}} .
\end{aligned}
$$

Since the right-hand side of Eq. 22 is real, and since the imaginary parts of the triple products are completely antisymmetric, we have

$$
\sum_{i, j, k} T_{i j k} p(i) p(j) p(k)=\sum_{i, j, k} \tilde{T}_{i j k} p(i) p(j) p(k),
$$

so we may consider just the real parts $\tilde{T}_{i j k}$. We obtain an equivalent expression for Eq. 22 in terms of the structure coefficients:

$$
\sum_{i, j, k} \tilde{S}_{i j k} p(i) p(j) p(k)=\frac{4}{d(d+1)^{2}} .
$$

Specializing to the case $d=3$, the pure states are described by probability vectors $\vec{p}$ that satisfy

$$
\begin{aligned}
\sum_{i} p(i)^{2} & =\frac{1}{6}, \\
\sum_{i, j, k} \tilde{S}_{i j k} p(i) p(j) p(k) & =\frac{1}{12} .
\end{aligned}
$$

Using the results in Sec. II , we find that the pure states for the canonical Hesse SIC are given by

$$
\begin{aligned}
\sum_{i} p(i)^{2} & =\frac{1}{6} \\
\sum_{i} p(i)^{3} & =\frac{1}{2} \sum_{(i j k) \in Q} p(i) p(j) p(k)
\end{aligned}
$$


where $Q$ is the set of index triples $(i j k)$ corresponding to the lines drawn in Fig. 1, where permutations of the indices $i, j, k$ are counted separately. Interestingly, Fig. 1 coincides with a combinatorial object called a finite affine plane of order 3 (which can also be identified with the unique 2-(9,3,1)-design) 26]. It contains 9 points and 12 lines, and the index triples in $Q$ correspond to any 3 points on the same line.

The pure states for any other qutrit SIC are located on the same sphere given by Eq. (25) but with a different set of values for $\tilde{S}_{i j k}$ in Eq. 24). If we substitute the values in Eq. (17) into Eq. (24), we obtain

$$
\frac{1}{2} \sum_{i} p(i)^{3}+\sum_{i \neq j \neq k} \tilde{S}_{i j k} p(i) p(j) p(k)=0 .
$$

\section{ORTHOGONAL TRANSFORMATIONS BETWEEN PROBABILITY VECTORS OF DISTINCT SICS}

In this section, we will show that, in any dimension, the probability vectors corresponding to two different SICs are related by an orthogonal transformation, a fact known from Ref. 27. We will then go on to show that in dimension 3 , the orthogonal transformation takes a remarkably simple form.

In arbitrary dimension $d$, consider a pair of distinct SICs with elements $\Pi_{i}^{\prime}$ and $\Pi_{j}$. In the vector space of operators, $\Pi_{i}^{\prime}$ and $\Pi_{j}$ correspond to the vertices of 2 identical regular simplices, which means they must be related by an orthogonal transformation. Formally, because a SIC forms a Hermitian basis in the space of operators, we can write

$$
\Pi_{i}^{\prime}=\sum_{j} R_{i j} \Pi_{j}
$$

Since every SIC element has unit trace, taking the trace on both sides of Eq. (30) gives us

$$
\sum_{j} R_{i j}=1
$$

Multiplying the left-hand side of Eq. (30) by $\Pi_{j}^{\prime}$ and the right-hand side by $\sum_{l} R_{j l} \Pi_{l}$, we have

$$
\begin{aligned}
\operatorname{Tr}\left(\Pi_{i}^{\prime} \Pi_{j}^{\prime}\right) & =\sum_{k, l} R_{i k} R_{j l}\left(\frac{d \delta_{k l}+1}{d+1}\right), \\
\Longrightarrow \delta_{i j} & =\sum_{k} R_{i k} R_{j k} .
\end{aligned}
$$

which confirms that $R_{i j}$ is indeed an orthogonal matrix. Using Eq. 32, it is now straightforward to show that

$$
p^{\prime}(i)=\sum_{j} R_{i j} p(j)
$$

Let $\Pi_{i}$ be the canonical Hesse SIC and let $\Pi_{i}^{(\eta \pm)}(t)$ be the SIC generated by the fiducial vector $\left|\psi_{t}^{(\eta \pm)}\right\rangle$. Let

$$
Q_{i}=\frac{1}{3}\left(4 \Pi_{i}-I\right)
$$

be the dual basis to $\Pi_{i}$ (so $\operatorname{Tr}\left(Q_{i} \Pi_{j}\right)=\delta_{i j}$ ). Then the orthogonal matrix which takes $\Pi_{i}$ onto $\Pi_{i}^{(\eta \pm)}(t)$ is

$$
R_{i j}^{(\eta \pm)}(t)=\operatorname{Tr}\left[\Pi_{i}^{(\eta \pm)}(t) Q_{j}\right]
$$

It turns out that the matrices $R^{(\eta \pm)}(t)$ have a very simple form. In the standard two-line notation, define the permutations

$$
\begin{aligned}
p^{(0+)} & =\left(\begin{array}{lllllllll}
1 & 2 & 3 & 4 & 5 & 6 & 7 & 8 & 9 \\
1 & 2 & 3 & 4 & 5 & 6 & 7 & 8 & 9
\end{array}\right), \\
p^{(0-)} & =\left(\begin{array}{llllllllll}
1 & 2 & 3 & 4 & 5 & 6 & 7 & 8 & 9 \\
1 & 3 & 2 & 4 & 6 & 5 & 7 & 9 & 8
\end{array}\right), \\
p^{(1+)} & =\left(\begin{array}{lllllllll}
1 & 2 & 3 & 4 & 5 & 6 & 7 & 8 & 9 \\
1 & 5 & 9 & 2 & 6 & 7 & 3 & 4 & 8
\end{array}\right), \\
p^{(1-)} & =\left(\begin{array}{lllllllll}
1 & 2 & 3 & 4 & 5 & 6 & 7 & 8 & 9 \\
1 & 9 & 5 & 2 & 7 & 6 & 3 & 8 & 4
\end{array}\right), \\
p^{(2+)} & =\left(\begin{array}{lllllllll}
1 & 2 & 3 & 4 & 5 & 6 & 7 & 8 & 9 \\
1 & 6 & 8 & 2 & 4 & 9 & 3 & 5 & 7
\end{array}\right), \\
p^{(2-)} & =\left(\begin{array}{lllllllll}
1 & 2 & 3 & 4 & 5 & 6 & 7 & 8 & 9 \\
1 & 8 & 6 & 2 & 9 & 4 & 3 & 7 & 5
\end{array}\right), \\
p^{(3+)} & =\left(\begin{array}{lllllllll}
1 & 2 & 3 & 4 & 5 & 6 & 7 & 8 & 9 \\
1 & 4 & 7 & 2 & 5 & 8 & 3 & 6 & 9
\end{array}\right), \\
p^{(3-)} & =\left(\begin{array}{lllllllll}
1 & 2 & 3 & 4 & 5 & 6 & 7 & 8 & 9 \\
1 & 7 & 4 & 2 & 8 & 5 & 3 & 9 & 6
\end{array}\right),
\end{aligned}
$$

Let $P^{(\eta \pm)}$ be the permutation matrix corresponding to $p^{(\eta \pm)}$, with matrix elements

$$
P_{i j}^{(\eta \pm)}=\delta_{j, p^{(\eta \pm)}(i)} .
$$

Also define

$$
\begin{gathered}
a(t)=\frac{1}{3}(1+2 \cos 2 t), \\
A(t)=\left(\begin{array}{ccc}
a(t) & a\left(t-\frac{\pi}{3}\right) & a\left(t+\frac{\pi}{3}\right) \\
a\left(t+\frac{\pi}{3}\right) & a(t) & a\left(t-\frac{\pi}{3}\right) \\
a\left(t-\frac{\pi}{3}\right) & a\left(t+\frac{\pi}{3}\right) & a(t)
\end{array}\right), \\
R(t)=\left(\begin{array}{ccc}
A(t) & 0 & 0 \\
0 & A(t) & 0 \\
0 & 0 & A(t)
\end{array}\right) .
\end{gathered}
$$

It is then straightforward, though somewhat tedious, to verify that

$$
R^{(\eta \pm)}(t)=\left[P^{(\eta \pm)}\right]^{-1} R(t) P^{(\eta \pm)} .
$$


Since $R(t)=I \otimes A(t)$, it follows that

$$
\operatorname{Det}\left[R^{(\eta \pm)}(t)\right]=\operatorname{Det}[R(t)]=\{\operatorname{Det}[A(t)]\}^{3} .
$$

Because $A(t)$ is a circulant matrix, its eigenvalues are given by

$$
\begin{aligned}
\lambda_{\ell} & =a(t)+\omega^{\ell} a\left(t-\frac{\pi}{3}\right)+\omega^{-\ell} a\left(t+\frac{\pi}{3}\right) \\
& =e^{2 i t \ell}
\end{aligned}
$$

for $\ell=-1,0,1$. This implies that $\operatorname{Det}[A(t)]=1$. Thus, $\operatorname{Det}\left[R^{(\eta \pm)}(t)\right]=1$ and $R^{(\eta \pm)}(t)$ is, in fact, a rotation matrix.

It is easily seen that

$$
R\left(t_{1}\right) R\left(t_{2}\right)=R\left(t_{1}+t_{2}\right), \quad R(0)=I .
$$

So the matrices $R(t)$ form a 1-parameter subgroup of the orthogonal group.

\section{THE BOUNDARY OF QUTRIT STATE SPACE}

A concrete way to understand the geometry of qutrit state space is to figure out what the convex body looks like. In this regard, we want to consider not just the pure states but all boundary points of the set. Some valuable insight into the shape of the boundary is gained by looking at the distance of the boundary states from the center of the space, the maximally mixed state $\rho=\frac{1}{d} I$ as a function of direction. Specifically, we can write the SIC probabilities in the form

$$
p(i)=\frac{1}{d^{2}}+r n(i)
$$

where $\vec{n}$ is a direction vector with

$$
\sum_{i} n(i)=0, \quad \sum_{i} n(i)^{2}=1 .
$$

Let $r(\vec{n})$ be the value of the $r$ corresponding to the quantum state on the boundary, which is given by Eq. 45. Here we calculate this function for the canonical Hesse SIC. Put differently, we are looking for the polar equation describing its boundary states.

The boundary is determined using the following lemma:

Lemma 1. Let $\rho$ be an arbitrary Hermitian operator on a 3-dimensional Hilbert space. Then

(i) $\rho$ is a density operator if and only if

$\operatorname{Tr}(\rho)=1, \quad \operatorname{Tr}\left(\rho^{2}\right) \leq 1, \quad 3 \operatorname{Tr}\left(\rho^{2}\right)-2 \operatorname{Tr}\left(\rho^{3}\right) \leq 1 ;$

(ii) $\rho$ is a density operator for a boundary state if and only if

$\operatorname{Tr}(\rho)=1, \quad \operatorname{Tr}\left(\rho^{2}\right) \leq 1, \quad 3 \operatorname{Tr}\left(\rho^{2}\right)-2 \operatorname{Tr}\left(\rho^{3}\right)=1 ;$ (iii) $\rho$ is a density operator for a pure quantum state if and only if

$$
\operatorname{Tr}(\rho)=1, \quad \operatorname{Tr}\left(\rho^{2}\right)=1, \quad 3 \operatorname{Tr}\left(\rho^{2}\right)-2 \operatorname{Tr}\left(\rho^{3}\right)=1 .
$$

Proof. We begin by proving necessity. Suppose $\rho$ is a density matrix. It immediately follows that $\operatorname{Tr}(\rho)=1$ and $\operatorname{Tr}\left(\rho^{2}\right) \leq 1$. To prove the remaining inequality, let $\alpha, \beta, 1-\alpha-\beta$ be the eigenvalues of $\rho$. We find

$$
3 \operatorname{Tr}\left(\rho^{2}\right)-2 \operatorname{Tr}\left(\rho^{3}\right)-1=-6 \alpha \beta(1-\alpha-\beta) \leq 0 .
$$

For $\rho$ to be a boundary state at least one of its eigenvalues must vanish, in which case

$$
3 \operatorname{Tr}\left(\rho^{2}\right)-2 \operatorname{Tr}\left(\rho^{3}\right)-1=0 .
$$

In addition, if $\rho$ is a pure state then $\operatorname{Tr}\left(\rho^{2}\right)=1$.

We now turn to the proof of sufficiency. Let $\rho=\rho^{\dagger}$ be such that

$$
\operatorname{Tr}(\rho)=1, \quad \operatorname{Tr}\left(\rho^{2}\right) \leq 1, \quad 3 \operatorname{Tr}\left(\rho^{2}\right)-2 \operatorname{Tr}\left(\rho^{3}\right) \leq 1 .
$$

The first equality means that we can take the eigenvalues of $\rho$ to be $\alpha, \beta, 1-\alpha-\beta$. From Eq. 47) we get

$$
\alpha \beta(1-\alpha-\beta) \geq 0 \text {. }
$$

Thus, either (i) all eigenvalues are non-negative or (ii) exactly two of them are negative. We can show that (ii) is impossible. Assume the contrary to hold. Without loss of generality $\alpha, \beta<0$, implying that $1-\alpha-\beta>1$, which in turn implies $\operatorname{Tr}\left(\rho^{2}\right)>1$, contrary to hypothesis. We conclude that $\rho$ is positive semi-definite, and consequently a density matrix.

Next assume that

$$
\operatorname{Tr}(\rho)=1, \quad \operatorname{Tr}\left(\rho^{2}\right) \leq 1, \quad 3 \operatorname{Tr}\left(\rho^{2}\right)-2 \operatorname{Tr}\left(\rho^{3}\right)=1 .
$$

Then,

$$
\alpha \beta(1-\alpha-\beta)=0,
$$

implying that at least one of the eigenvalues must be zero. So $\rho$ is on the boundary of state space.

Finally assume

$$
\operatorname{Tr}(\rho)=1, \quad \operatorname{Tr}\left(\rho^{2}\right)=1, \quad 3 \operatorname{Tr}\left(\rho^{2}\right)-2 \operatorname{Tr}\left(\rho^{3}\right)=1 .
$$

Using the argument above the eigenvalues are $0, \alpha, 1-\alpha$. Since $\operatorname{Tr}\left(\rho^{2}\right)=1$ it must be that $\alpha=0$ or 1 and therefore $\rho$ is a rank-1 projection operator.

We can use the lemma for the quantum states associated with the canonical Hesse SIC. To this end, recall its structure coefficients in Eq. (19). We use these to calculate $\operatorname{Tr}\left(\rho^{2}\right)$ and $\operatorname{Tr}\left(\rho^{3}\right)$ for $\rho$ given by Eq. 22. We find that

$$
\begin{aligned}
& \operatorname{Tr}\left(\rho^{2}\right)=12 \sum_{i} p(i)^{2}-1, \\
& \operatorname{Tr}\left(\rho^{3}\right)=1+24 \sum_{i} p(i)^{3}-12 \sum_{(i j k) \in Q} p(i) p(j) p(k),
\end{aligned}
$$




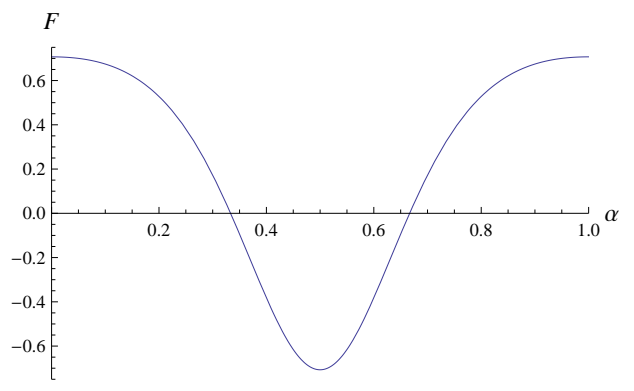

FIG. 2. A plot of $F$ as a function of one of the eigenvalues $\alpha$ for boundary states.

where $Q$ is again the set of lines on the affine plane in Fig. 1. Substituting Eq. (45) into the probabilities above, we obtain

$$
\begin{aligned}
\sum_{i} p(i)^{2} & =\frac{1}{9}+r^{2} \\
\sum_{i} p(i)^{3} & =\frac{1}{81}+\frac{r^{2}}{3}+r^{3} \sum_{i} n(i)^{3}, \\
\sum_{(i j k) \in Q} p(i) p(j) p(k) & =\frac{8}{81}-\frac{r^{2}}{3}+r^{3} \sum_{(i j k) \in Q} n(i) n(j) n(k) .
\end{aligned}
$$

Consequently, we can restate the conditions in Lemma 1 as follows:

(i) $\rho$ is a density operator if and only if

$$
r^{2} \leq \frac{1}{18}, \quad 4 r^{3} F(\vec{n})-r^{2}+\frac{1}{54} \geq 0 ;
$$

(ii) $\rho$ is a density operator on the boundary of the state space if and only if

$$
r^{2} \leq \frac{1}{18}, \quad 4 r^{3} F(\vec{n})-r^{2}+\frac{1}{54}=0 ;
$$

(iii) $\rho$ is a density operator for a pure state if and only if

$$
r^{2}=\frac{1}{18}, \quad F(\vec{n})=\frac{1}{\sqrt{2}}
$$

where

$$
F(\vec{n})=\sum_{i} n(i)^{3}-\frac{1}{2} \sum_{(i j k) \in Q} n(i) n(j) n(k) .
$$

Thus, the value of $r(\vec{n})$ giving the distance of a boundary state from the completely mixed state along the direction of $\vec{n}$ is the smallest positive root of

$$
4 r^{3} F(\vec{n})-r^{2}+\frac{1}{54}=0 .
$$

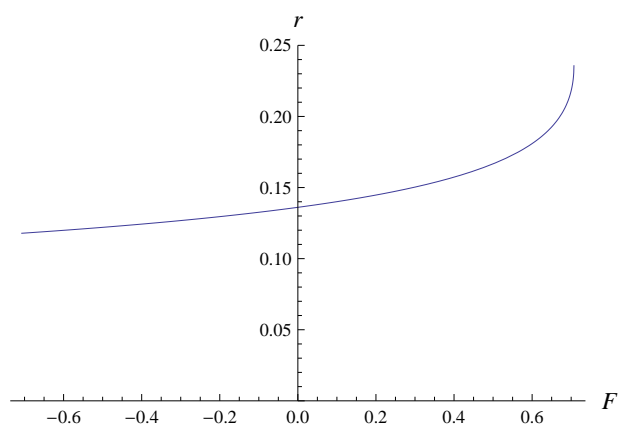

FIG. 3. The radial distance $r$ of boundary states from the maximally mixed state, as a function of $F=F(\vec{n})$.

To determine the bounds on $F \equiv F(\vec{n})$, we can write it in terms of the eigenvalues of $\rho$ by performing some algebra on Eq. (54) and Eq. (55). Denoting the eigenvalues of $\rho$ by $\alpha, \beta$, and $1-\alpha-\beta$, we obtain

$$
F=\frac{\sqrt{3}\left(\frac{2}{9}-f_{1}+f_{2}\right)}{\left(f_{1}-\frac{1}{3}\right)^{3 / 2}}
$$

where $0 \leq \alpha, \beta, 1-\alpha-\beta \leq 1$ and

$$
\begin{aligned}
& f_{1}=\alpha^{2}+\beta^{2}+(1-\alpha-\beta)^{2}, \\
& f_{2}=\alpha^{3}+\beta^{3}+(1-\alpha-\beta)^{3} .
\end{aligned}
$$

From Eq. 61), it can easily be shown that

$$
-\frac{1}{\sqrt{2}} \leq F \leq \frac{1}{\sqrt{2}}
$$

where the upper (respectively, lower) bound is achieved when two of the eigenvalues are identical and $<\frac{1}{3}$ (respectively, $>\frac{1}{3}$ ). If all the eigenvalues are equal to $\frac{1}{3}$ this corresponds to the maximally mixed state, for which $F$ is undefined. For boundary states, at least one of the eigenvalues must be zero. So the only case we need to consider for $F$ is when $\beta=1-\alpha$. Figure 2 shows $F$ as a function of $\alpha$, provided that one of the eigenvalues vanishes.

In terms of $F$, the desired root in Eq. 60 is given by

$$
r= \begin{cases}\frac{1}{12 F}\left(1+\frac{g}{\omega^{\sigma}}+\frac{\omega^{\sigma}}{g}\right) & \text { if } F \neq 0, \\ \frac{1}{3 \sqrt{6}} & \text { if } F=0,\end{cases}
$$

where $\sigma \equiv \operatorname{sgn}(F)$ and $g$ is the cube root with the smallest positive argument in

$$
g^{3}=1-4 F^{2}+2 F \sqrt{4 F^{2}-2} .
$$

When $F= \pm \frac{1}{\sqrt{2}}, g=e^{i \frac{\pi}{3}}$ and we get the bounds for $r$ :

$$
\frac{1}{6 \sqrt{2}} \leq r \leq \frac{1}{3 \sqrt{2}}
$$


A plot of $r$ as a function of $F$ is shown in Fig. 3

Finally let us note that we can use this method to answer other questions about the geometry of the set of qutrits. For instance, it has been shown [28, 29] that the 8-dimensional ball $r \leq \frac{1}{3 \sqrt{2}}$ is truncated by the 7dimensional faces of the probability simplex. It is interesting to ask about the pure states located on these faces. To answer this question, consider, for example, the 7-dimensional face with center point $\vec{c}$ given by $c(i)=\frac{1}{8}$ for $i \neq 9$ and $c(9)=0$. In this case, we can take probability vectors of the form

$$
p(i)=\frac{1}{8}+s m(i), \quad p(9)=m(9)=0,
$$

where

$$
\sum_{i=1}^{8} m(i)=0, \quad \sum_{i=1}^{8} m(i)^{2}=1 .
$$

Going through a similar argument as the one above, we find that the states on the face are given by $s=0$, or

$$
s^{2} \leq \frac{1}{24}, \quad F(\vec{m})=\frac{3}{16 s}[2-\Phi(\vec{m})]
$$

where

$$
\begin{aligned}
\Phi(\vec{m})= & {[m(1)+m(5)]^{2}+[m(2)+m(4)]^{2} } \\
& +[m(3)+m(6)]^{2}+[m(7)+m(8)]^{2}
\end{aligned}
$$

and $F$ is the same function defined in Eq. (59). In particular, the pure states correspond to $\vec{m}$ such that

$$
F(\vec{m})=\frac{3 \sqrt{6}}{8}[2-\Phi(\vec{m})] .
$$

\section{SUMMARY AND OUTLOOK}

With the recent revival of interest in addressing foundational issues in quantum theory, we pose a simple yet intriguing question: What is the shape of the set of quantum states $\mathcal{C} \subset \mathbb{R}^{d^{2}}$ ? Our preliminary attempt to address this question revolves around a description of quantum states in terms of the outcome probabilities of a SICPOVM. This particular representation allows us to exploit the intrinsic symmetry of a SIC in mapping density operators to probability vectors, which we believe not only serves as a natural "coordinate system" for studying the underlying geometry of quantum states, but also provides us with an interpretation of quantum states in terms of Bayesian probabilities 30.

In this work, we focused our attention on $d=3$, which is the simplest, nontrivial case to examine. We considered the infinitely many Weyl-Heisenberg qutrit SICs, which are classified into SIC-families corresponding to orbits of the Clifford group. Each SIC can be uniquely identified with a set of complex numbers called triple products $T_{i j k}$, the trace of the product of three SIC elements, whose polar angles are related to discrete geometric phases [31, 32 and to Bargmann invariants 33. in complex projective space, and whose imaginary parts give SICs the structure of a Lie algebra [19]. We also have structure coefficients $S_{i j k}$, which are the expansion coefficients when multiplying SIC projectors, and whose real parts $\tilde{S}_{i j k}$ are especially convenient for describing geometric properties of qutrits.

Using $\tilde{S}_{i j k}$ for the canonical Hesse SIC given by $\left|\psi_{0}\right\rangle$ in Eq. (12), we discovered the most economical description for SIC probability vectors associated with qutrit pure states, which are given by Eq. (27) and Eq. 28). Studying the probabilities for the canonical Hesse SIC is sufficient because we demonstrated that the probabilities for other qutrit SICs are related to it by a 9dimensional rotation that can be expressed in terms of a single function - $a(t)$ in Eq. (38).

The remarkable simplicity of Eq. 287 suggests that the geometric structure of qutrits is largely determined by the symmetries associated with a finite affine plane. For example, observe that if we consider the indices as points in Fig. 1 the permutations given in Eq. (36) are such that they preserve the affine lines. Also, using the notion of maximal consistent sets in Ref. [28, a set of probability vectors associated with the Hesse configuration of vectors on a Hilbert space can be maximized into a convex body that has the same largest inscribed sphere and smallest containing sphere as qutrit state space, and also shares some of its 2-dimensional sections. Therefore, to gain a proper understanding of the convex geometry of qutrits, it is crucial to understand the full significance of Eq. (28).

We also described a polar equation for the qutrit boundary. We found that the function $F(\vec{n})$ that yields the radial distance of a boundary state in direction $\vec{n}$ from the uniform distribution is the same function that picks out the pure states for the canonical Hesse SIC, i.e., $F(\vec{p})=0$ for any $\vec{p}$ on the sphere containing pure states, which again highlights the important role played by the finite affine plane of Fig. 1 .

Lastly, it is our hope that the results presented here also serve as evidence for the utility of the SIC representation in matters regarding quantum foundations.

\section{ACKNOWLEDGMENTS}

The authors are grateful to Chris Fuchs and Åsa Ericsson for useful discussions. This work was supported in part by the U.S. Office of Naval Research (Grant No. N00014-09-1-0247). DMA was also supported in part by the John Templeton Foundation. Research at Perimeter Institute is supported by the Government of Canada through Industry Canada and by the Province of Ontario through the Ministry of Research and Innovation. 
[1] G. Kimura, Phys. Lett. A 314, 339 (2003).

[2] M. S. Byrd and N. Khaneja, Phys. Rev. A 68, 062322 (2003).

[3] I. Mendaš, J. Phys. A: Math. Gen. 39, 11313 (2006).

[4] S. K. Goyal, B. N. Simon, R. Singh, and S. Simon, arXiv:1111.4427.

[5] G. Sarbicki and I. Bengtsson, J. Phys. A: Math. Theor. 46, 035306 (2013).

[6] G. Zauner, Quantendesigns. Grundzüge einer nichtkommutativen Designtheorie, Ph.D. thesis, Univ. Wien (1999), translated to English in Int. J. Quant. Inf. 9, 445 (2011).

[7] C. M. Caves, Symmetric Informationally Complete POVMs, internal report (University of New Mexico, 1999).

[8] J. M. Renes, R. Blume-Kohout, A. J. Scott, and C. M. Caves, J. Math. Phys. 45, 2171 (2004).

[9] D. M. Appleby, J. Math. Phys. 46, 052107 (2005).

[10] S. Weigert, Int. J. Mod. Phys. B 20, 1942 (2006).

[11] C. Godsil and A. Roy, Euro. J. Combin. 30, 246 (2009).

[12] A. J. Scott and M. Grassl, J. Math. Phys. 51, 042203 (2010).

[13] C. A. Fuchs and R. Schack, Found. Phys. 41, 34556 (2011).

[14] C. A. Fuchs, Quant. Inf. Comput. 4, 467 (2004).

[15] D. Gottesman, Phys. Rev. A 57, 127 (1998).

[16] D. M. Appleby, arXiv:0909.5233 (2009).

[17] I. R. Porteous, Clifford Algebras and the Classical Groups
(Cambridge University Press, Cambridge, 1995).

[18] H. Zhu, J. Phys. A: Math. Theor. 43, 305305 (2010).

[19] D. M. Appleby, S. T. Flammia, and C. A. Fuchs, J. Math. Phys. 52, 022202 (2011).

[20] L. P. Hughston, "d=3 SIC-POVMs and Elliptic Curves," Seminar at the Perimeter Institute. Available at http://pirsa.org/07100040/ (2007).

[21] I. Bengtsson, J. Phys. Conf. Ser. 254, 012007 (2010).

[22] M. Krein and D. Milman, Stud. Math. 9, 133 (1940).

[23] S. T. Flammia, (2004), unpublished.

[24] N. S. Jones and N. Linden, Phys. Rev. A 71, 012324 (2005).

[25] D. M. Appleby, H. B. Dang, and C. A. Fuchs, arXiv:0707.2071.

[26] J. W. P. Hirschfeld, Projective Geometries over Finite Fields (Oxford University Press, Oxford, 1998).

[27] S. N. Filippov and V. I. Man'ko, J. Russ. Laser Res. 31, 211 (2010).

[28] D. M. Appleby, A. Ericsson, and C. A. Fuchs, Found. Phys. 41, 564 (2011).

[29] J. I. Rosado, Found. Phys. 41, 1200 (2011).

[30] C. A. Fuchs, arXiv:1003.5209 (2010).

[31] M. V. Berry, Proc. R. Soc. Lond. A 392, 45 (1984).

[32] Y. Aharonov and J. Anandan, Phys. Rev. Lett. 58, 1593 (1987).

[33] V. Bargmann, J. Math. Phys. 5, 862 (1964). 